\title{
Online Health Searching: A Perspective from South East Asia
}

\section{ISSN: 2637-7748}

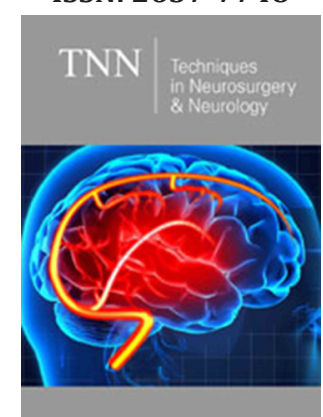

*Corresponding author: Anushia Inthiran, University of Canterbury, New Zealand

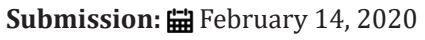

Published: 笽February 19, 2020

Volume 3 - Issue 2

How to cite this article: Anushia Inthiran. Online Health Searching: A Perspective from South East Asia. Tech Neurosurg Neurol.3(2). TNN.000559.2020. DOI: 10.31031/TNN.2020.03.000559

Copyright@ Anushia Inthiran, This article is distributed under the terms of the Creative Commons Attribution 4.0 International License, which permits unrestricted use and redistribution provided that the original author and source are credited.

\author{
Anushia Inthiran* \\ University of Canterbury, New Zealand
}

Opinion

Searching for online health information is an activity conducted by people from all walks of life. Performing a health search is not a trivial task. A searcher could be overwhelmed by the sheer amount of information available and the need to understand technical and complex details; more so when the search is performed by a layperson. However, the complexity and technicality of performing an online health search does not discourage health consumers from performing a health search on the Internet. Research studies from developed countries provide information on the type of person who performs the search, sources used, type of search performed, and devices used to perform the search. However, similar information from South East Asia is limited. There are many reasons for this, among them being the lack of medical knowledge and experience and low levels of health literacy in comparison to health consumers in the developed world. Nevertheless, there is some information about online health information searching from South East Asia. In recent years, researchers have started shedding light on the online health information searching behaviour of health consumers from South East Asia. Often, the purpose of these studies is to explore, describe and compare online health searching behaviour of health consumers in developing countries and in developed countries. These research studies conducted in South East Asia contribute to literature within this field and has made the online health information searching scene rich. The aim of this opinion piece is to describe what is known about the online health information searching behaviour within the South East Asian region and to postulate direction of online health searching within this region. South East Asian online health consumers indicate experiencing positive and negative search experience. In some cases, post search outcomes influenced the search experience. Results of a study indicate information overload as the main negative concern. Other issues include pages that contained too much information, pages were not properly structured and presence of too many links on the page. This contributed to the search process being cumbersome [1]. Results also indicate that the search process influenced participants emotionally and resulted in them limiting the search activity from proceeding further. As a coping mechanism some participants question the comments and arguments presented on the site whilst other ignored any serious implications presented on the page [2].

This suggests that the structure of the page and the 'look and feel' of the page influenced the search experience. This means that issues in relation to human computer interaction and interface design needs to be paid attention too when developing websites. Whilst in the past, technical content may have been the main reason for a negative search experience, it has become apparent that now-setup, design and structure of a page is equally important. South East Asian health consumers are also influenced emotionally when performing a health search; and on the hindsight, the progression of a health search may change depending on what information is found and how information found is perceived. Another aspect that is interesting amongst South East Asian consumers is the source used to perform the online health search. For example, one would expect that because of low knowledge and health literacy, a health consumer may end of up searching on a simpler or generic website. However, this is not the case in urban areas of South East Asia. Results of a study conducted in urban areas indicate sites like MedlinePlus, Medline, The Mayo Clinic, The National Institute of Health 
Website (NIH), The Johns Hopkins University Website and WebMD are utilised. These are highly medical sites that possibly require a higher level of knowledge and understanding [1]. This suggests that whilst some online health consumers are concerned with design and structure of a page, others are more concerned about obtaining higher level understanding and that too from reputable websites. The last aspect that is explored is the utilisation or consumption of information. Current studies indicate that South East Asian consumers use online health information found in a variety of ways, but information sharing seems to be the most popular utilisation technique. Often information is shared with the participant's partner being husband or wife; followed by sharing information with participant's sibling. In the case of sibling participants always shared information with a female sibling; a younger or/and older sister [3-6]. Sharing is specifically done electronically first and this is followed by a face to face conversation. However, interesting enough, whilst information is shared, the search process is always conducted as a solo effort. One reason as to why searching for health information conducted as a solo effort is because search process is dominated by the better searcher. This led to the other partner being left out of the search process and taking on the role of a passive collaborator. Another interesting point to note is that sharing of health information is a private activity with selected family members. It is noted that a female sibling is the only family member with whom information is shared with. This could be attributed to the role of womenfolk in SEA which is to primarily take care of the family whereas men are breadwinners.

Another research study probed the possibility of online health consumers sharing information with the doctor. Interestingly, results indicate participants mostly discussed information found with the doctor. However, the way information was discussed fell into two categories. The first category being elementary conversation and the second - guarded conversation. In the elementary conversation category, participants indicate minimal communication. In the guarded category participants were cautions about approaching the subject on having found information online. In this category, conversation about information found went on for longer than just the exchange of several sentences. The aim of the conversation was to seek clarification and obtain opinion. In summary, whilst the general level of online health information searching in the South East Asian region is at its infancy, results of research studies indicate that the initial evaluation is positive. The activity of searching for health information is performed, generic and medical type websites are used. Whilst most online health consumers have mixed feelings about the search experience, this situation is the same even in the developed world. The aspect of sharing information is one to be lauded as this enables knowledge creation and sharing allowing health consumers to take charge of one's health as this region progresses towards patient centred healthcare and share decision making.

\section{References}

1. Inthiran A, Alhashmi SM, PK Ahmed (2016) Online consumer health: A Malaysian perspective. New Zealand.

2. Information Technology in Developing Countries, London, UK.

3. Inthiran A (2016) Online health search experience: sentiments from south East Asia. International Journal of Knowledge Content Development \& Technology 6(2): 29-42.

4. Inthiran A (2017) Information sharing and search collaboration activities of health consumers in South East Asia. Proceedings of the 2017 Conference on Conference Human Information Human Information Interaction, pp. 254-248.

5. Inthiran A (2017) Doctor-patient communication of health information found online: Preliminary results from South East Asia.

6. International Conference on Asian Digital Libraries, pp. 307-313. 